Type of the Paper (Article.)

\title{
Analytical and Semi-Analytical Solutions for the Self and Mutual Inductances of Concentric Coplanar Ordinary and Bitter Disk Coils
}

Slobodan Babic ${ }^{1, *}$

1 Independent Researcher; e-slobobob@yahoo.com

* Correspondence: slobobob@yahoo.com; 53 Berlioz, 101, H3E 1N2, Montreal (Qc), Canada

\begin{abstract}
In this paper, closed and semi-closed form solutions are presented for the self - and mutual inductance of ordinary and Better disk coils which lie concentrically in a plane. The solutions are given as the combination of the elliptic integral of the second kind and a simple integral or only as the elliptic integral of the second kind. All formulas or obtained in remarkably simple form and give extremely accurate results with significantly neglectable computational time. All cases either regular or singular (disks in contact or overlap) are covered. The formulas for the mutual inductances can be directly used to calculating the self-inductance of the ordinary disk coil or the Bitter disk coil, respectively. Many presented examples show the excellent numerical agreement with previous publish methods.
\end{abstract}

Keywords: Ordinary disk coils; Bitter disk coils; mutual inductance, self-inductance. 


\section{Introduction}

Several monographs and papers are devoted to calculating the self and the mutual inductance for the ordinary circular coils (massive coils of the rectangular cross-section, thin wall solenoids, disk coils) with the azimuthal current density [1-12]. The conventional coils used in many applications such as all ranges of transformers, generators, motors, current reactors, magnetic resonance applications, antennas, coil guns, medical electronic devices, superconducting magnets, tokamaks, electronic and printed circuit board design, plasma science, etc., are very well-known. Also, there are circular coils with the radial current density (massive coils of the rectangular cross-section, disk coils) which are interesting from an engineering aspect. These coils are the well-known Bitter coils [13-18] which supply extremely high magnetic fields up to $45 \mathrm{~T}$. There are other applications such as the homopolar motors, homopolar generators, and Faraday's disk. The thin disk coils (pancakes) are of the particular interest in previously mentioned electromagnetic applications. In [8] J.T. Conway propose the analytical solutions for the self- and mutual Inductances of ordinary concentric coplanar disk coils. He gives the excellent solution as generalized hypergeometric functions which are closely related to elliptic integrals. The method used is a Legendre polynomial expansion of the inductance integral, which renders all integrations straightforward. In this paper we give remarkably simple solutions for calculating the self- and mutual inductance of the ordinary concentric coplanar and the Bitter disk coils. In the case of the ordinary concentric coplanar disks the solutions are obtained in semi-analytical and analytical form. The solutions are obtained over the elliptic integral of the second kind $E(k)$ and one simple integral whose kernel function is integrable over all interval of integration. In the case of the concentric coplanar Bitter disk coils the solutions are obtained in the analytical form over the elliptic integral of the second kind $E(\mathrm{k})$. All cases given in [8] are verified and confirmed by the presented approach. Also, the self- and the mutual inductance of the concentric coplanar disk coils are verified by the filament method [15-18]. All obtained results are in an excellent agreement.

\section{Basic Expressions}

2.1 Ordinary concentric coplanar disks

The mutual inductance between two ordinary coplanar disks is given by [8],

$$
M=\frac{\mu_{0} N_{1} N_{2}}{\left(R_{2}-R_{1}\right)\left(R_{4}-R_{3}\right)} \int_{0}^{\pi} \int_{R_{1}}^{R_{2}} \int_{R_{3}}^{R_{4}} \frac{r_{1} r_{2} \cos (\theta) d r_{1} d r_{2} d \theta}{\sqrt{r_{1}^{2}-2 r_{1} r_{2} \cos (\theta)+r_{2}^{2}}}
$$

where,

$N_{1}$ and $N_{2}$ are equally spaced turns of disks,

$R_{1}$ and $R_{3}$ are inner radii of disks (m),

$R_{2}$ and $R_{4}$ are outer radii of disks $(\mathrm{m})$,

$\mu_{0}=4 \pi \cdot 10^{-7}(\mathrm{H} / \mathrm{m})$

The self-inductance of a disk coil with equally spaced turns $N$ and $R_{1}$ and $R_{2}$ inner and outer radii and, respectively, is given by [8],

$$
L=\frac{\mu_{0} N^{2}}{\left(R_{2}-R_{1}\right)^{2}} \int_{0}^{\pi} \int_{R_{1}}^{R_{2}} \int_{R_{1}}^{R_{2}} \frac{r_{1} r_{2} \cos (\theta) d r_{1} d r_{2} d \theta}{\sqrt{r_{1}^{2}-2 r_{1} r_{2} \cos (\theta)+r_{2}^{2}}}
$$

In (1) and (2) $r_{1}$ and $r_{2}$ are the cilindrical coordinates.

\subsection{Concentric coplanar Bitter disks}

The mutual inductance between two Bitter disks is given by [17],

$$
M_{B}=\frac{\mu_{0} N_{1} N_{2}}{\ln \frac{R_{2}}{R_{1}} \ln \frac{R_{4}}{R_{3}}} \int_{0}^{\pi} \int_{R_{1}}^{R_{2}} \int_{R_{3}}^{R_{4}} \frac{\cos (\theta) d r_{1} d r_{2} d \theta}{\sqrt{r_{1}^{2}-2 r_{1} r_{2} \cos (\theta)+r_{2}^{2}}}
$$


and the self-inductance of the Bitter disk coil is given by [17],

$$
L_{B}=\frac{\mu_{0} N^{2}}{\left[\ln \frac{R_{2}}{R_{1}}\right]^{2}} \int_{0}^{\pi} \int_{R_{1}}^{R_{2}} \int_{R_{3}}^{R_{4}} \frac{\cos (\theta) d r_{1} d r_{2} d \theta}{\sqrt{r_{1}^{2}-2 r_{1} r_{2} \cos (\theta)+r_{2}^{2}}}
$$

\section{Calculation Method}

3.1. Ordinary concentric coplanar disks

Before the first integration let us take the following substitution $\theta=\pi-2 \beta$ so that (1) becomes,

$$
M=\frac{-2 \mu_{0} N_{1} N_{2}}{\left(R_{2}-R_{1}\right)\left(R_{4}-R_{3}\right)} \int_{0}^{\pi / 2} \int_{R_{1}}^{R_{2}} \int_{R_{3}}^{R_{4}} \frac{r_{1} r_{2} \cos (2 \beta) d r_{1} d r_{2} d \beta}{\sqrt{r_{1}^{2}+2 r_{1} r_{2} \cos (2 \beta)+r_{2}^{2}}}
$$

The first integration in (5), [19-20] gives:

$$
I_{r_{1}}=\int_{R_{1}}^{R_{2}} \frac{r_{1} d r_{1}}{\sqrt{r_{1}^{2}+2 r_{1} r_{2} \cos (2 \beta)+r_{2}^{2}}}=\left\{\sqrt{r_{1}^{2}+2 r_{1} r_{2} \cos (2 \beta)+r_{2}^{2}}-r_{2} \cos (2 \beta) \operatorname{asinh} \frac{r_{1}+r_{2} \cos (2 \beta)}{r_{2} \sin (2 \beta)}\right\}_{R_{1}}^{R_{2}}
$$

The second integration in (5), [19-20] gives:

$$
\begin{aligned}
& I_{r_{2}}=\int_{R_{3}}^{R_{4}} r_{2} I_{r_{1}} d r_{2}=\frac{1}{3} \sum_{n=1}^{n=4}(-1)^{n-1}\left\{\left(l_{n}^{2}+\rho_{n}^{2}\right) \sqrt{l_{n}^{2}+2 l_{n} \rho_{n} \cos (2 \beta)+\rho_{n}^{2}}-\right. \\
& \left.-l_{n}^{3} \cos (2 \beta) a \sinh \frac{\rho_{n}+l_{n} \cos (2 \beta)}{l_{n} \sin (2 \beta)}-\rho_{n}^{3} \cos (2 \beta) a \sinh \frac{l_{n}+\rho_{n} \cos (2 \beta)}{\rho_{n} \sin (2 \beta)}\right\}
\end{aligned}
$$

where,

$$
\rho_{1}=\rho_{2}=R_{1}, \rho_{3}=\rho_{4}=R_{2}, l_{1}=l_{4}=R_{3}, l_{2}=l_{3}=R_{4}
$$

The third integration in (5), [19-20] gives:

$$
\begin{aligned}
& I_{3}=\int_{0}^{\pi / 2} \cos (2 \beta) I_{r_{2}} d \theta=\frac{1}{6} \sum_{n=1}^{n=4}(-1)^{n-1}\left\{l_{n} \rho_{n}\left(l_{n}+\rho_{n}\right) E\left(k_{n}\right)-\right. \\
& \left.-\int_{0}^{\pi / 2} l_{n}^{3} a \sinh \frac{\rho_{n}+l_{n} \cos (2 \beta)}{l_{n} \sin (2 \beta)} d \beta-\int_{0}^{\pi / 2} \rho_{n}^{3} a \sinh \frac{l_{n}+\rho_{n} \cos (2 \beta)}{\rho_{n} \sin (2 \beta)} d \beta\right\}
\end{aligned}
$$

Finally, the mutual inductance between two coplanar ordinary disks is,

$$
M=\frac{\mu_{0} N_{1} N_{2}}{3\left(R_{2}-R_{1}\right)\left(R_{4}-R_{3}\right)} \sum_{n=1}^{n=4}(-1)^{n}\left\{l_{n} \rho_{n}\left(l_{n}+\rho_{n}\right) E\left(k_{n}\right)-\right.
$$

$\left.I_{n}\right\}$

(6) where,

$$
\begin{gathered}
I_{n}=\int_{0}^{\pi / 2}\left\{l_{n}^{3} a \sinh \frac{\rho_{n}+l_{n} \cos (2 \beta)}{l_{n} \sin (2 \beta)}+\rho_{n}^{3} a \sinh \frac{l_{n}+\rho_{n} \cos (2 \beta)}{\rho_{n} \sin (2 \beta)}\right\} d \beta \\
k_{n}^{2}=\frac{4 \rho_{n} l_{n}}{\left(\rho_{n}+l_{n}\right)^{2}} \\
\rho_{1}=\rho_{2}=R_{1}, \rho_{3}=\rho_{4}=R_{2}, l_{1}=l_{4}=R_{3}, l_{2}=l_{3}=R_{4}
\end{gathered}
$$

$E(k)$ - elliptic integral of the second kind [19-20]. 
The mutual inductance is obtained in remarkably simple form over the elliptic integral of the second kind and one simple integral whose kernel function is the continue and integrable over the domain of the integration so that (6) is applicable in the regular or the singular cases (disks are in the contact or overlap).

For calculating the self-inductance of the ordinary disk coil one can use (2) or directly putting in (6) $R_{1}=R_{3}$, $R_{2}=R_{4}$ and $N_{1}=N_{2}=N$ that gives,

$$
L=\frac{2 \mu_{0} N^{2}}{3\left(R_{2}-R_{1}\right)^{2}}\left\{\left(R_{1}^{3}+R_{2}^{3}\right)(2 G-1)+R_{2} R_{1}\left(R_{2}+R_{1}\right) E(k)-\right.
$$

$\left.I_{0}\right\}$

where,

$$
\begin{gathered}
I_{0}=\int_{0}^{\pi / 2}\left\{R_{1}^{3} a \sinh \frac{R_{2}+R_{1} \cos (2 \beta)}{R_{1} \sin (2 \beta)}+R_{2}^{3} a \sinh \frac{R_{1}+R_{2} \cos (2 \beta)}{R_{2} \sin (2 \beta)}\right\} d \beta \\
k^{2}=\frac{4 R_{1} R_{2}}{\left(R_{1}+R_{2}\right)^{2}}
\end{gathered}
$$

$G=0.91596559417721901505460351493238411 \ldots$, Catalan'sconstant

We put $R_{2}=\alpha R_{1}(\alpha>1)$ and (7) becomes,

$$
L=\frac{2 \mu_{0} N^{2} R_{1}}{3(\alpha-1)^{2}}\left\{\left(\alpha^{3}+1\right)(2 G-1)+\alpha(\alpha+1) E(k)-\right.
$$

$\left.I_{0}(\alpha)\right\}$

where,

$$
\begin{gathered}
I_{0}=\int_{0}^{\pi / 2}\left\{\operatorname{asinh} \frac{\alpha+\cos (2 \beta)}{\sin (2 \beta)}+\alpha^{3} \operatorname{asinh} \frac{1+\alpha \cos (2 \beta)}{\alpha \sin (2 \beta)}\right\} d \beta \\
k^{2}=\frac{4 \alpha}{(\alpha+1)^{2}}
\end{gathered}
$$

From (2) or (7) it is possible to obtain the self-inductance of the full disk $\left(R_{1}=0\right.$ and $\left.R_{2}=R\right)$ as follows,

$$
L=\frac{2 \mu_{0} N^{2} R_{1}}{3}(2 G-
$$

1)

\subsection{Concentric coplanar Bitter disks}

Before the first integration let us take the following substitution $\theta=\pi-2 \beta$ so that (3) becomes,

$$
M_{B}=\frac{-2 \mu_{0} N_{1} N_{2}}{\ln \frac{R_{2}}{R_{1}} \ln \frac{R_{4}}{R_{3}}} \int_{0}^{\pi} \int_{R_{1}}^{R_{2}} \int_{R_{3}}^{R_{4}} \frac{\cos (2 \beta) d r_{1} d r_{2} d \beta}{\sqrt{r_{1}^{2}+2 r_{1} r_{2} \cos (2 \beta)+r_{2}^{2}}}
$$

The first integration in (10), [19-20] gives:

$$
I_{r_{1}}=\int_{R_{1}}^{R_{2}} \frac{d r_{1}}{\sqrt{r_{1}^{2}+2 r_{1} r_{2} \cos (2 \beta)+r_{2}^{2}}}=\left\{\operatorname{asinh} \frac{r_{1}+r_{2} \cos (2 \beta)}{r_{2} \sin (2 \beta)}\right\}_{R_{1}}^{R_{2}}
$$


The second integration in (10), [19-20] gives:

$$
I_{r_{2}}=\int_{R_{3}}^{R_{4}} I_{r_{1}} d r_{2}=\sum_{n=1}^{n=4}(-1)^{n-1}\left\{l_{n} a \sinh \frac{\rho_{n}+l_{n} \cos (2 \beta)}{l_{n} \sin (2 \beta)}+\operatorname{asinh} \frac{l_{n}+\rho_{n} \cos (2 \beta)}{\rho_{n} \sin (2 \beta)}\right\}
$$

where.

$$
\rho_{1}=\rho_{2}=R_{1}, \rho_{3}=\rho_{4}=R_{2}, l_{1}=l_{4}=R_{3}, l_{2}=l_{3}=R_{4}
$$

The third integration in (10), [19-20] gives:

$$
I_{3}=\int_{R_{3}}^{R_{4}} \cos (2 \beta) I_{r_{2}} d \theta=\sum_{n=1}^{n=4}(-1)^{n-1}\left(l_{n}+\rho_{n}\right) E\left(k_{n}\right)
$$

Finally, the mutual inductance between two coplanar Bitter disks is,

$$
M_{B}=
$$

$\frac{2 \mu_{0} N_{1} N_{2}}{\ln \frac{R_{2}}{R_{1}} \ln \frac{R_{4}}{R_{3}}} \sum_{n=1}^{n=4}(-1)^{n}\left(l_{n}+\rho_{n}\right) E\left(k_{n}\right)$

where,

$$
\begin{gathered}
k_{n}^{2}=\frac{4 \rho_{n} l_{n}}{\left(\rho_{n}+l_{n}\right)^{2}} \\
\rho_{1}=\rho_{2}=R_{1}, \rho_{3}=\rho_{4}=R_{2}, \quad l_{1}=l_{4}=R_{3}, l_{2}=l_{3}=R_{4}
\end{gathered}
$$

The mutual inductance is obtained in amazingly simple form over the elliptic integral of the second kind. It is applicable in the regular or the singular cases (disks are in the contact or overlap).

For calculating the self-inductance of the Bitter disk coil one can use (4) or directly putting in (6) $R_{1}=R_{3}, R_{2}=R_{4}$ and $N_{1}=N_{2}=N$ that gives,

$$
L_{B}=\frac{4 \mu_{0} N^{2}\left(R_{1}+R_{2}\right)}{\left[\ln \frac{R_{2}}{R_{1}}\right]^{2}}[E(k)-
$$

1]

where,

$$
k^{2}=\frac{4 R_{1} R_{2}}{\left(R_{1}+R_{2}\right)^{2}}
$$

Thus, either the mutual inductance or the self-inductance for the Bitter Disks are obtained in the close form over the elliptic integral of the second kind.

\section{Numerical Validation}

Example 1.

In [8] Conway give the calculation of normalized self-inductance in terms of the shape factor $R_{2}==\alpha R_{1}$ as $L_{N}=$ $L / N^{2} / R_{1}$, (Table 1$)$.

Table 1. Self-inductance given as $L_{N}=L / N^{2} / R_{1}$ in $\mu \mathrm{H} / \mathrm{m}$ 


\begin{tabular}{|c|c|c|c|}
\hline$\alpha$ & {$[4]$} & {$[8]$ Equ-(58) } & This work (8) \\
\hline 1.5 & 3.9375 & 3.937556957309482 & 3.937556957309482 \\
\hline 3 & 4.1202 & 4.120247770949786 & 4.120247770949786 \\
\hline 4 & 4.6535 & 4.653592975459867 & 4.653592975459867 \\
\hline 7 & 6.5440 & 6.544182694169381 & 6.544182694169381 \\
\hline 9 & 7.8795 & 7.879438694940537 & 7.879438694940537 \\
\hline 19 & 14.740 & 14.73964824153050 & 14.73964824153050 \\
\hline 39 & 28.628 & 28.62677689946369 & 28.62677689946369 \\
\hline
\end{tabular}

Table 1 shows the comparison between inductance given by Kalantarov [4] and calculated in [8] with (58) as well as with (8) from this work. The agreement is excellent in all cases.

\section{Example 2.}

Table 2 shows calculations of the self-inductance of the ordinary disk for various shape factors and for values remarkably close to the logarithmic singularity as $\alpha \rightarrow 1$. These are compared to two sets of results given by Spielrein method [5] and [8] with (58).

Table 2. Comparison of calculations for the self-inductance for values of the shape factor both close and far from the singularity $\alpha \rightarrow 1$ Results are in $\mu \mathrm{H} / \mathrm{m}$.

\begin{tabular}{|c|c|c|c|}
\hline$\alpha$ & {$[5]$} & {$[8]$ Equ-(58) } & This work (8) \\
\hline 50 & 36.2822050627 & 36.28220506268449 & 36.28220506268449 \\
\hline 10 & 8.5558078657 & 8.555807865723495 & 8.555807865723494 \\
\hline 3 & 4.1202478984 & 4.120247770949786 & 4.120247770949786 \\
\hline 1.5 & 3.9375565536 & 3.937556957309482 & 3.937556957309482 \\
\hline 1.1 & 5.1875898298 & 5.187589829874826 & 5.187589829874826 \\
\hline 1.01 & 7.8169836166 & 7.816983616632973 & 7.816983616632973 \\
\hline 1.001 & 10.6712873756 & 10.67128737563754 & 10.67128737563754 \\
\hline 1.00001 & 16.4524421475 & 16.45244214746880 & 16.45244214746880 \\
\hline 1.000001 & 19.3458776688 & 19.34587766869611 & 19.34587766869611 \\
\hline 1.0000001 & 22.2393823064 & 22.23938230721058 & 22.23938230721058 \\
\hline
\end{tabular}

Again, the excellent agreement of this work (8) with (58) in [1] and very well close with [5]. Equation (8) gives much better accurate results (for $\alpha-1 \rightarrow 10^{-5}, 10^{-6}, \ldots$ ) then (3) in [12].

Example 3.

In this example we show the performance of the formula (8) when the singularity is approached $\left(\alpha-1 \rightarrow 10^{-16}\right)$. We compare the results of the formula (8) with those obtained by the asymptotic formula (20) and (58) in [8], Table 3.

Table 3. Comparison of (8) and (58), [8] with asymptotic formula (20), [8] as the singularity at unit shape factor isapproached. Results are in $\mu \mathrm{H} / \mathrm{m}$.

\begin{tabular}{|c|c|c|c|}
\hline$\alpha-1$ & {$[8]$ Equ-(20) } & [8] Equ-(58) & This work (8) \\
\hline $10^{-1}$ & 4.998068846886786 & 5.187589829874826 & 5.187589829874826 \\
\hline
\end{tabular}




\begin{tabular}{|l|l|l|l|}
\hline $10^{-2}$ & 7.784316262102376 & 7.816983616632973 & 7.816983616632973 \\
\hline $10^{-3}$ & 10.66658208170639 & 10.67128737563754 & 10.67128737563754 \\
\hline $10^{-6}$ & 19.34586862407991 & 19.34587766869611 & 19.34587766869611 \\
\hline $10^{-8}$ & 25.13289490994222 & 25.13289502932351 & 25.13289502932351 \\
\hline $10^{-10}$ & 30.91992242743389 & 30.91992242891705 & 30.91992242891705 \\
\hline $10^{-12}$ & 36.70694995724185 & 36.70694995725958 & 36.70694995725958 \\
\hline $10^{-15}$ & 45.38749125213915 & 45.38749125213918 & 45.38749125213918 \\
\hline $10^{-16}$ & 48.28100501710534 & 48.28100501710534 & 48.28100501710534 \\
\hline
\end{tabular}

All results are in remarkably good agreement. Thus, the formula (8) has the large range of application.

\section{Example 4.}

Calculate the self-inductance of the full disk coil for which $R=1(\mathrm{~m})$ and $N=100$.

From (9) the self-inductance of the full disk is,

$$
L_{\text {Full-Disk }}=6.969570425670744(\mathrm{mH})
$$

To verify this result we use $(7)$ and $R_{1}=10^{-17}(\mathrm{~m}), R_{2}=1(\mathrm{~m}), N=100$ that gives,

$$
L_{\text {Full-Disk }}=6.969570425670744(\mathrm{mH})
$$

We obtain the identical result.

From previous examples we show that the self-inductance (8) is applicable in large range of $\alpha$.

Thus, remarkably simple formula (8) can be used as the general formula for calculating the self-inductance of the ordinary disk coil (pancake).

\section{Example 5.}

Table 4 gives the calculations of the mutual inductance in millihenrys for two disk coils where the radii and are given the fixed values: $R_{2}=1(\mathrm{~m}), R_{3}=0.4(\mathrm{~m})$, and $R_{4}=0.6(\mathrm{~m})$, whereas $R_{1}$

varies between 0.1 and $0.9 \mathrm{~m}$. The turn numbers and are both specified to be 100, [8]. This covers all the generic overlap cases.

Table 4. Mutual inductance in $\mathrm{mH}$ and CPU in seconds for all overlap cases.

\begin{tabular}{|c|c|c|c|c|}
\hline$R_{1}(m)$ & $\begin{array}{c}M_{12}(m H) \\
{[8]}\end{array}$ & $\begin{array}{c}\text { CPU } \\
(\mathrm{sec})[8]\end{array}$ & $\begin{array}{c}M_{12}(m H) \\
(6) \text { This work }\end{array}$ & $\begin{array}{c}\text { CPU (sec) } \\
\text { This work }\end{array}$ \\
\hline 0.1 & 8.3452226401271722326 & 2.262 & 8.3452226401271722326 & 0.0 \\
\hline 0.2 & 9.2662149362835422651 & 2.215 & 9.2662149362835422651 & 0.0 \\
\hline 0.3 & 10.178800706150685814 & 1.778 & 10.178800706150685814 & 0.0 \\
\hline 0.4 & 10.751261394420867987 & 1.092 & 10.751261394420867987 & 0.0 \\
\hline
\end{tabular}




\begin{tabular}{|l|c|c|c|c|c|}
\hline 0.5 & 10.014392107083652030 & 1.373 & 10.014392107083652030 & 0.0 \\
\hline 0.6 & 8.2517781770123374536 & 0.094 & 8.2517781770123374536 & 0.0 \\
\hline 0.7 & 7.1724760612076564190 & 0.140 & 7.1724760612076564190 & 0.0 \\
\hline 0.8 & 6.5028431844372044556 & 1.109 & 6.5028431844372044556 & 0.0 \\
\hline 0.9 & 6.0061106298597996959 & 0.094 & 6.0061106298597996959 & 0.0 \\
\hline
\end{tabular}

Table 4 shows that all results are identical, obtained by [9] and (6) from this work but the CPU times by this work is practically zero. In Mathematica programing [21] using the instruction 'Timing' the computational time is 0 seconds. Using the instruction 'Absolute Timing' the computational time is $3 \cdot 10^{-7}$ seconds. We confirmed that relatively simple equation (6) covers all overlap cases for the negligible computation time and with the excellent accuracy

Example 6.

Calculate the mutual inductance between two disk coils for which is, $R_{1}=1(\mathrm{~m}), \quad R_{2}=2(\mathrm{~m}), R_{3}=1(\mathrm{~m}), R_{4}=2(\mathrm{~m})$ and $N_{1}=N_{2}=N=1000$.

Obviously, the disks completely overlap it is the case of the self-inductance. Let us apply equation (6) for the mutual inductance of the disk coils. It gives,

$$
M=L=3.794801607308613(\mathrm{H})
$$

Applying the formula (7) for the self-inductance of the disk coil we obtain,

$$
L=3.794801607308613
$$

We obtain the identical result.

Thus, we show that the simple formula (6) is general for any case either for the regular or for the singular. It is confirmed in the previous examples.

\section{Example 7.}

Table 5 gives the calculations of mutual inductance in millihenrys for two disk coils where the radii and are given the fixed values: $R_{2}=10(\mathrm{~m}), R_{3}=4(\mathrm{~m})$, and $R_{4}=6(\mathrm{~m})$, whereas $R_{1}$ varies between 1 and $9 \mathrm{~m}$. The turn numbers and are both specified to be 1000 . This covers all the overlap cases.

Table 5. Mutual inductance in $\mathrm{H}$ for all overlap cases.

\begin{tabular}{|c|c|c|}
\hline$R_{1}(m)$ & This work $(11) M_{B}(H)$ & $M_{B}(H)[14-15]$ \\
\hline 1 & 6.176953480899784 & 6.176890611593179 \\
\hline 2 & 8.442109820575922 & 8.441929573292805 \\
\hline 3 & 10.32822091283136 & 10.32810057022684 \\
\hline 4 & 11.43930901799091 & 11.44063208654759 \\
\hline 5 & 10.40642626084792 & 10.40633203780527 \\
\hline 6 & 8.263044997910772 & 8.263044761562908 \\
\hline 7 & 7.051548638612127 & 7.051548623257631 \\
\hline 8 & 6.338568884058207 & 6.338568878810852 \\
\hline
\end{tabular}


From Table 5 we can see the very god agreement of all results. There is the negligible discrepancy between presented results because the filament method [14-15] is approximative and depends on the number of the divisions of the disk coils. Many disk divisions are related with the computational time.

\section{Example 8.}

Calculate the self-inductance of the Bitter disk coil using equations (1) and (12).

The disk dimensions and the number of turns is as follows, $R_{1}=1(\mathrm{~m}), R_{2}=2(\mathrm{~m}), N=1000$.

Equation (11) calculate the mutual inductance between two Bitter disk coils. Let us take $R_{1}=1(\mathrm{~m}), R_{2}=2(\mathrm{~m}), R_{3}=$ $1(m), R_{4}=2(m), N_{1}=N_{2}=1000$. .Applying (11) we obtain,

$$
M_{B}=L_{B}=3.569912886724817(H)
$$

Applying the formula for the self-inductance of the Bitter coil we have,

$$
L_{B}=3.569912886724817(H)
$$

Thus, we obtain the same expected results.

\section{Conclusion}

In this paper we presented remarkably simple solutions for calculating the self - and mutual inductance of the ordinary concentric coplanar and the Bitter disk coils. All cases either the regular or the singular (disks overlap) are cover with obtained formulas in the semi-analytical and analytical form. We show that with formulas for the mutual inductance is possible to obtained directly results for the corresponding self-inductances. The presented formulas are much familiar and easier to use for the potential readers that those given in [8]. In [8] are given more complicated functions with which more people are not familiar. In this work all calculations are made by Mathematica [21]. From presented examples one can see that all compared results are practically identical or in the excellent agreement.

Author Contributions: Conceptualization, methodology, software, validation, formal analysis, investigation, resources, writing - original draft preparation, writing - review and editing, visualization.

Funding: This research received no external funding.

Conflicts of Interest: The author declares no conflict of interest.

\section{References}

1. Grover, F.W. Inductance Calculations; Dover: New York, NY, USA, 1964.

2. Dwight, H.B. Electrical Coils and Conductors; McGraw-Hill Book Company, Inc.: New York, NY, USA, 1945.

3. Snow, C. Formulas for Computing Capacitance, and Inductance; National Bureau of Standards Circular 544: Washington, DC, USA, December 1954. 
4. P. L. Kalantarov, Inductance Calculations. Moscow, Russia: National Power Press, 1955.

5. J. Spielrein, “Die Induktivität eisenfreier Kreisringspulen,” Arch. El., vol. 3, p. 187, 1915.

6. S. Butterworth, "On the self-inductance of single-layer flat coils," in Proc. Phys. Soc., London,

U.K., Dec. 1919, vol. 32, pp. 31-37.

7. E. B. Rosa and F. W. Grover, Scientific Papers of the National Bureau of Standards, 3rd ed.

Washington, DC, USA: National Bureau of Standards, 1948.

8. J. T. Conway, “Analytical Solutions for the Self- and Mutual Inductances of Concentric

Coplanar Disk Coils," IEEE Trans. Magn., vol. 49, no. 3, pp. 1135-1142, Mar. 2013.

9. J. T. Conway, “Analytical and Semi-Analytical Solutions for the Force Between Circular Loops in Parallel Planes," IEEE Trans. Magn., vol. 49, no. 8, pp. 4817-4823, August 2013.

10. J. T. Conway, “Forces Between Thin Coils with Parallel Axes Using Bessel Functions," IEEE Trans. Magn., vol. 49, no. 9, pp. 5028-5034, Sep. 2013.

11. S. Babic, S. Salon, C. Akyel, “ The mutual inductance of two thin coaxial disk coils in air," IEEE Trans. Magn., vol. 42, no. 2, pp. 822-825, Mar. 2013.

12. S. I. Babic and C. Akyel, "An improvement in the calculation of the self-inductance of thin disk coils with air-core," WSEAS Trans. Circuits Syst., vol. 3, no. 8, pp. 1621-1626, Oct. 2004.

13. Bitter, F.: “The design of powerful electromagnets part II. The magnetizing coil,” Rev. Sci. Instrum., 1936, 7, (12), pp. 482-489.

14. Ren, Y.; Wang, F.; Kuang, G.; Chen, W.; Tan, Y.; Zhu, J.; He, P., “Mutual Inductance and Force Calculations between Coaxial Bitter Coils and Superconducting Coils with Rectangular Cross Section," J. Supercond. Nov. Magn. 2010, 24, pp. 1687-1691.

15. Ren, Y.; Kuang, G.; Chen, W., “ Inductance of Bitter Coil with Rectangular Cross-section,” J. Supercond. Nov. Magn. 2012, 26, pp. 2159-2163.

16. Conway, J.T, “Non coaxial force and inductance calculations for bitter coils and coils with uniform radial current distributions," In Proceedings of the 2011 International Conference on Applied Superconductivity and Electromagnetic Devices, Sydney, NSW, Australia, 14-16, December 2011.

17. S. I. Babic and C. Akyel," Mutual inductance and magnetic force calculations for coaxial Bitter disk coils (Pancakes)," IET Science, Measurement \& Technology, vol. 10, no. 8, pp. 972-976, Nov. 2016.

18 S. I. Babic and C. Akyel, "Self-Inductance of the Circular Coils of the Rectangular Cross-Section with the Radial and Azimuthal Current Densities," Physics, MDPI, vol. 2, no. 3, pp. 352-367, July 2020.

19 I. S. Gradshteyn and I. M. Ryzhik, Table of Integrals, Series and Products, 7th ed. New York, NY, USA: Academic, 2007.

20 A. P. Prudnikov, Yu. A. Brychkov, and O. I. Marichev, Integrals and Series. New York, NY, USA: Gordon and Breach, 1990, vol. 3.

21.S. Wolfram, The Mathematica Book, 5th ed. Champaign, IL, USA: Wolfram Media, 2003. 